\title{
Anesthetic Management of a Child with Mucopolysaccharidosis: A Case Report
}

\author{
Dr. Jigyasa Shahani ${ }^{1}$, Dr. Neelam Dogra ${ }^{2}$ \\ Assistant Professor, Department of Anesthesiology, SMS Medical College \& Attached Hospitals, Jaipur, India
}

\begin{abstract}
Mucopolysaccharidosis (MPS) is a group of genetic disorders that presents challenges during anesthetic care and in particular difficulty with airway management. We describe the anaesthetic management of a seven month old child with Hurler syndrome, who presented for an inguinal hernia repair.
\end{abstract}

Keywords: Mucopolysaccharidosis, Hurler's syndrome, difficult intubation, anesthesia

\section{Introduction}

Mucopolysaccharidoses (MPS) are a group of rare genetic lysosomal storage disorders characterized by the deficiency or complete lack of necessary lysosomal enzymes required for the stepwise breakdown of glycosaminoglycans (GAGs). ${ }^{1-5}$ Consequently, fragments of GAGs accumulate intracellularly in the lysosome resulting in cellular enlargement causing dysfunction of structure and function of tissues. This process leads to numerous clinical abnormalities. This group of diseases are known to cause major problems in anaesthesia. We describe the anaesthetic management of an infant with MPS I ( Hurler's syndrome).

\section{Case Report}

A seven months male child with mucopolysaccharidoses (Hurler's syndrome) was scheduled for right sided inguinal herniotomy. He weighed $6 \mathrm{kgs}$. The child was prone to frequent respiratory infections. His developmental milestones were delayed. He used to snore during sleep and preferred to sleep in prone position. He had normal intelligence for age. He had coarse facies, macroglossia and a short neck. Clinical examination of the cardiovascular and respiratory systems did not reveal any abnormality. Haemogram, urine examination and liver function tests were within normal limits. Echocardiography showed a thickened mitral valve with mild mitral regurgitation. Aortic, pulmonary and tricuspid valves were normal. No regional wall motion abnormalities were seen. Mild kyphotic abnormality at dorsolumbar junction was present. His airway assessment revealed normal mouth opening, a thick and large tongue with a modified Mallampati Grade IV view.

Pre-emptive caudal analgesia with general anaesthesia for repair of the inguinal hernia was planned. In preanaesthesia room during IV access, his skin was found to be dry, thick and rough. ECG, NIBP and SpO2 monitors were attached.Sedative premedication was avoided and glycopyrrolate $0.004 \mathrm{mg} / \mathrm{kg} \quad$ I.V. was administered. Antibiotic was administered for bacterial endocarditis prophylaxis. An appropriate sized laryngeal mask airway and equipment for transtracheal jet ventilation were kept at hand. Anaesthesia was induced with sevoflurane in 100\% oxygen by facemask. Laryngoscopy using straight blade was done in deeper plane with the patient breathing
spontaneously.Laryngeal structures were not visible even after posterior displacement of laryngeal cartilages. Repeated attempts were made ensuring oxygen saturation \& at the final attempt when laryngoscope blade introduced through left angle of mouth, tip of the epiglottis was seen markedly shifted to left side. Cricoid pressure by the assistant improved the laryngeal view with the posterior extremity of glottis barely visible and a $3.5 \mathrm{~mm}$ portex uncuffed orotracheal tube was inserted with help of stylet. Correct placement of the tracheal tube was confirmed by capnography. ETT was connected to Jackson-Rees circuit and manual ventilation started. Anesthesia was then maintained with oxygen, nitrous oxide and halothane. Muscle paralysis was achieved with atracurium $3 \mathrm{mg}$. Before surgery, the child was placed in the lateral position and caudal block was performed with $6 \mathrm{ml}$ of $0.2 \%$ ropivacaine. The child maintained normal vital signs throughout the surgery. Inguinal hernia was repaired and surgery lasted for $30 \mathrm{~min}$. Residual effects of neuromuscular blocking agent was antagonized with neostigmine and glycopyrrolate. The trachea was extubated uneventfully when the child was fully awake. The post-operative period was unremarkable and the child was discharged on the third post-operative day.

\section{Discussion}

Hurler syndrome or MPS $1 \mathrm{H}$ is the prototype of MPS and is the most severe form of it. ${ }^{2}$ In Hurler's syndrome airway problem has been described as the worst in pediatric anesthesia. ${ }^{1}$ Perioperative mortality rates averaging 20\% have been reported for patients with this disease, with failure to control the airway as the largest single cause of mortality. ${ }^{6}$ MPS are rare conditions, incidence varying from 1 in 24,000-5, 00,000 population. ${ }^{7}$ Multiple factors are present in the MPS to make airway management and tracheal intubation potentially hazardous. ${ }^{1}$ In addition to generalized infiltration and thickening of the soft tissues, the oropharynx may be obstructed by a large tongue with or without tonsillar hypertrophy. Chronic infection further narrows the airway and results in the presence of copious thick secretions. The neck is typically short and immobile and cervical spine and temporomandibular joint may have a limited range of movement. The trachea is often narrow and flattened. ${ }^{1}$ Chest deformity along with deposits in lower respiratory tract and lung interstitium may cause obstructive lung disease and diffusion defects leading to hypercapnia, 


\section{International Journal of Science and Research (IJSR) \\ ISSN (Online): 2319-7064 \\ Index Copernicus Value (2013): 6.14 | Impact Factor (2014): 5.611}

hypoxia and elevated airway pressures. ${ }^{4,8}$ They are also likely to develop myocardial hypertrophy, ventricular dysfunction, cardiomyopathy and heart failure following pulmonary hypertension. ${ }^{3,7}$ Nerve and tendon entrapment is common in them. Due to deposition of mucopolysaccharides in brain cells they may develop progressive mental retardation of varying degree. Thickening of meninges may lead to hydrocephalus and hypertrophic cervical pachymeningitis that may result in myelopathy associated with nerve root compression. ${ }^{3}$ The uniqueness of their anatomy and extremely sensitive airway often result in failed intubation and bronchospasm even after successful intubation. Premedicants cannot be counted upon to produce a sedated and cooperative patient because of dangers of upper airway obstruction, respiratory depression, hypercarbia and cardiorespiratory arrest. An antisialogogue is essential in view of copious airway secretions. Bacterial endocarditis prophylaxis with antibiotics is recommended for all MPS patients with valvular cardiac lesions. ${ }^{7}$ Intraperitoneal procedures, will require general endotracheal anaesthesia to provide a secure upper airway for controlled ventilation during neuromuscular paralysis and to protect abnormal lungs from aspiration of gastric contents. ${ }^{7}$ A selection of endotracheal tubes is needed since age adjusted and anatomic formulae for endotracheal tube size do not apply to patients with MPS due to unique configurations of the laryngeal inlet and subglottic area. A technique for airway management and tracheal intubation utilizing the brain laryngeal mask airway, the fibreoptic bronchoscope, a guide wire and a ureteral dilator in children with mucopolysaccharidoses has been described. ${ }^{9}$ An anaesthetic sequence which ensures early return of consciousness and airway reflexes is recommended. Postoperative management of all children with MPS should include continued airway management until fully conscious, and monitoring to detect episodes of airway obstruction and desaturation. High dependency care is ideal. ${ }^{1}$

Children with MPS present the anaesthesiologist with a tremendous challenge. A full knowledge of the pathophysiology of this group of diseases is essential in order to be fully aware of the implications of submitting a child to anaesthesia and surgery.

\section{Reference}

[1] Walker RW, Daroskim, Morris P, Wraith JE: Anaesthesia and mucopolysaccharidoses. A review of airway problems in children. Anaesthesia; 1994, 49:1078-84.

[2] Baines D, Keneallay J: Anaesthetic implications of the mucopolysaccharidoses: a fifteen year experience in a children's hospital. Anaesth Intensive Care; 1983, 11:198-202.

[3] Herrick IA, Rhine EJ: The mucopolysaccharidoses and anaesthesia: a report of clinical experience. Can $J$ of Anaesth; 1988, 35:67-73.

[4] Neufeld EF, Muenzer J, et al: The mucopolysaccharidoses. In: Scriver C, Beaudet A, Sly $\mathrm{W}$, et al., editors. The metabolic and molecular bases of inherited disease. 8. New York: McGraw-Hill; 2001, pp. 3421-3452.
[5] Wappner RS: Lysosomal Storage Disorders. In: McMillan JA, Feigin RD, et al., editors. Oski's Pediatrics Principles and practice. 4. Pennsylvania: JB Lippincott Co, 2006, pp. 2199-2217.

[6] Wilder RT, Belani KG. Fiberoptic intubation complicated by pulmonary edema in a 12 year old child with Hurler syndrome. Anaesthesiology 1990; 72; 20507.

[7] 7.Diaz JH, Belani KG. Perioperative management of children with Mucopolysaccharidosis. Anesth Analg 1993; 77: 1261-70.

[8] Bartz HJ, Wiesner L, Wappler F. Anesthetic management of patients with Mucopolysaccharidosis IV presenting for major orthopedic surgery. Acta Anaesthesiol Scand 1999; 43: 679-83.

[9] Walker RW, Allen DL, Rothera MR. A fibreotic intubation technique for children with mucopolysaccharidoses using the laryangeal mask airway. Paediatr Anaesth 1997; 7: 42 